\title{
Multiple odontogenic abscesses. Thoracic and abdomino-perineal extensión in an immuno competent patient
}

\author{
Bartolomé Arias-Chamorro ${ }^{1}$, Marian Contreras-Morillo ${ }^{2}$, Antonio Acosta-Moyano ${ }^{3}$, Francisco Ruiz-Delga- \\ do ${ }^{4}$, Lucas Bermudo-Añino ${ }^{5}$, Antonio Valiente-Álvarez ${ }^{6}$
}

\author{
${ }^{1}$ Maxillofacial Surgery Resident, Hospital Carlos Haya, Malaga, Spain \\ ${ }^{2}$ Maxillofacial Surgery Resident, Hospital Carlos Haya, Malaga, Spain \\ ${ }^{3}$ Maxillofacial Surgery Staff, Hospital Carlos Haya, Malaga, Spain \\ ${ }^{4}$ Maxillofacial Surgery Staff, Hospital Carlos Haya, Malaga, Spain \\ ${ }^{5}$ Head of Management Unit, Maxillofacial Surgery Service, Hospital Carlos Haya, Malaga, Spain \\ ${ }^{6}$ Head of Maxillofacial Surgery Service, Hospital Carlos Haya, Malaga, Spain
}

Correspondence:

C/Recogidas $n^{\circ} 491^{\circ}$ derecha

CP: 18002 Granada, Spain

bartarias@hotmail.com

Received: 21/02/2010

Accepted: 09/04/2010

\begin{abstract}
Arias-Chamorro B, Contreras-Morillo M, Acosta-Moyano A, Ruiz-Delgado F, Bermudo-Añino L, Valiente-Álvarez A. Multiple odontogenic abscesses. Thoracic and abdomino-perineal extensión in an immuno competent patient. Med Oral Patol Oral Cir Bucal. 2011 Sep 1;16 (6):e772-5. http://www.medicinaoral.com/medoralfree01/v16i6/medoralv16i6p772.pdf
\end{abstract}

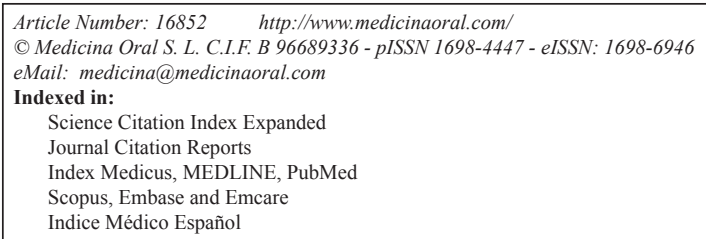

Indice Médico Español

\begin{abstract}
Introduction: Odontogenic infection (OI) may lead to death if it extends beyond the buccal area. The virulence of pathogens and the local and systemic status of the patient influence the propagation of the pathogen, either by anatomical continuity or haemematogenous dissemination. Several severe complications derived from OI have been reported in the head, neck and chest. However, OI with an abdominal component, caused by bacteraemia with dental foci or the direct passage of pus from the thorax to the abdomen, are unusual.

Case report: We present the case of a young immunocompetent woman who, after false cure of an odontogenic abscess, again reported gynaecological symptoms. A network of connected abdomino-perineal, thoracic and cervical abscesses was discovered.

Discussion: The peculiarity and severity of this case is a reminder that treatment of an abscessed OI should include intravenous broad-spectrum antibiotics, together with surgical drainage of the purulent collections. Samples should be taken for culture and an antibiogram in order to use specific antibiotics if the initial empirical therapy shows resistance. The diagnosis and follow-up should be by CT, which in our patient showed anatomical continuity of the abscesses from the dental focus. The time sequence of the symptoms, in the absence of any other infectious cause, revealed the descending odontogenic nature of the process.
\end{abstract}

Key words: Focal dental infection, mediastinitis, vulvar vestibulitis, pelvic infection, periapical abscess, periapical disease. 


\section{Introduction}

Odontogenic infection (OI) is that which arises from the teeth or periodontal tissues. OI are usually localized and self-limiting, though they may sometimes spread and even become very severe. Propagation can be produced by continuity or by lymphatic or haematogenous dissemination and depends on the patient's local and systemic factors and on the virulence of the pathogen. Multiple severe complications of OI have been reported, such as Ludwig's angina, thoracic empyema, mediastinitis, necrotizing fasciitis, cavernous sinus thrombosis, cerebral abscess, meningitis and septicaemia (1). Reports can also be found of sinusitis and maxillary osteomyelitis, orbital abscess, abscess causing airway compression, abscess of the carotid sheath and jugular thrombophlebitis, pleuritis and other processes related with the bacteraemia, the toxaemia caused by the pathogens, the induced autoimmune response and dissemination / abscessification via the anatomical planes $(2,3)$, as the routes of least resistance. There are three potential routes descending from the neck to the mediastinum by which an OI may progress: the retropharyngeal / retroesophageal route, which is the most important; and the perivascular and the pretracheal routes (4).

The few reports of an OI with an abdominal component are normally related with bacteraemia from dental foci, and include liver (5), suprarenal (6) and retroperitoneal (7) abscesses, and occasionally abscesses due to continuity via the diaphragm following anatomical planes $(4,8)$. We present a case of vulvar-perineal abscess forming part of a network of abdominal, thoracic and cervical collections of pus that followed a downward course from a dental focus.

\section{Case Report}

A 21-year-old woman with no medical history of note presented to the emergency department with a two-day history of pain in molar 47 and tumefaction of the right submandibular region, which had not been treated previously. Her vital signs were normal, she had no fever and a blood test showed 20,000 leukocytes ( $89 \%$ neutrophils). An orthopantomography and cervical ultrasound were reported as a suspected $4 \times 3 \mathrm{~cm}$ sublingual abscess near the midline. A contrast-enhanced orocervical CT showed a hypointense $2 \times 1.6 \mathrm{~cm}$ image with a phlegmonous aspect slightly enlarging the airway (Fig. 1). The patient was admitted to the maxillofacial ward for parenteral medical treatment (amoxicillin-clavulanic acid $1 \mathrm{~g} / 8 \mathrm{~h}+$ gentamycin $240 \mathrm{mg} / 24 \mathrm{~h}+$ methylprednisolone $80 \mathrm{mg} / 8 \mathrm{~h}$ ) and surgical drainage. The following day she underwent exodoncy (complex due to the limited buccal opening) of tooth 47 , with abundant outpouring of pus through the alveolus. Her course was uneventful with clear clinical improvement, and no cervical drainage or further $\mathrm{CT}$ were performed. One

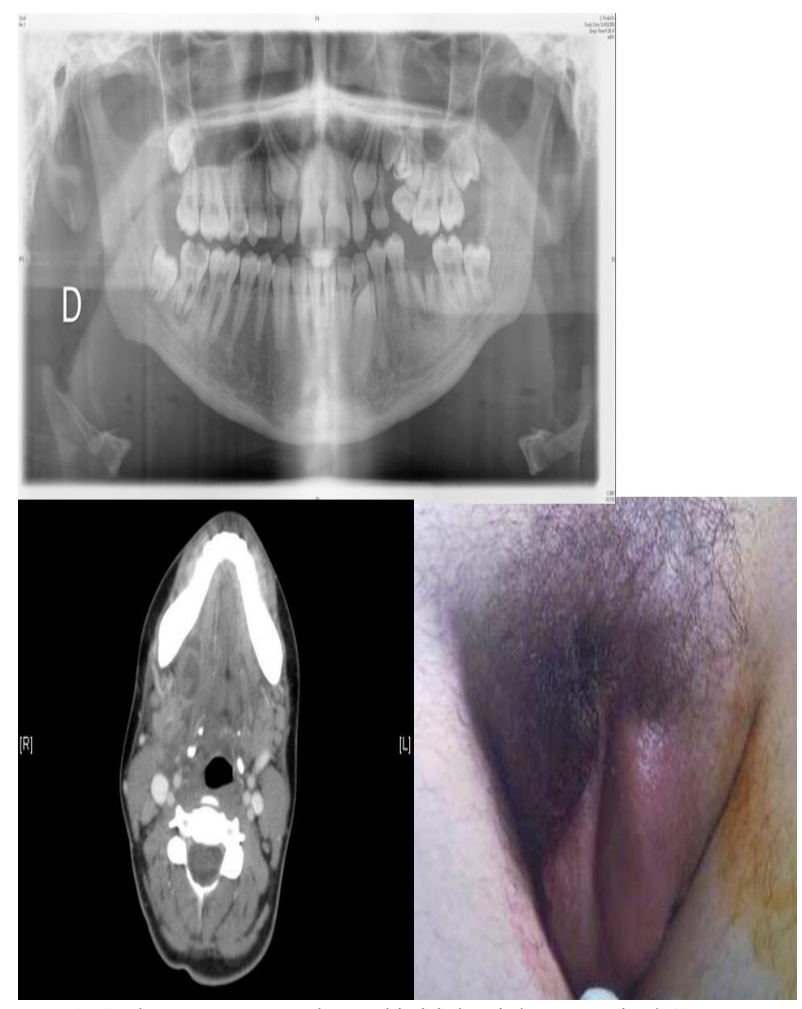

Fig. 1. Orthopantomography and initial axial orocervical CT. Image of the vulvitis at the second admission.

week later the patient was discharged with oral antibiotic treatment (amoxicillin-clavulanic acid $875 \mathrm{mg} / 8 \mathrm{~h}$ ).

Five days later she was readmitted urgently to the gynaecology service with a vulvar abscess (Fig. 1). Surgical drainage was performed under general anaesthesia of the left labia majora and mons venus. Drainage tubes were left in place and samples taken for culture and an antibiogram.

Empirical antibiotic treatment was again started with i.v. clindamycin $600 \mathrm{mg} / 8 \mathrm{~h}$, i.v. ampicilin $1 \mathrm{~g} / 8 \mathrm{~h}$ and i.v. gentamycin $240 \mathrm{mg} / 24 \mathrm{~h}$. The culture results showed colonies of Escherichia coli, Bacterioides and Streptococcus mitis. After 3 days on the ward with fever of up to $39^{\circ} \mathrm{C}$, abundant drainage of purulent material, laboratory alterations (leukocytes 49000, platelets 128000 , prothrombin time of $66 \%$, creatinin 1.8 , albumin 0.9 , NA 131, K 2.9) and hypotension (70/30) she was transferred to the ICU with a diagnosis of sepsis, where her clinical condition worsened. Examination showed left pulmonary hypoventilation and hepatosplenomegaly, with no signs of peritoneal irritation, with left flank pain and the vulva with important tumefaction. Chest radiography showed pleural effusion and laminar atelectasia in the base of the left lung. The antibiotic treatment was changed to i.v. piperacillin and tazobactam 4 g/ 6 h, i.v. amikacin $500 \mathrm{mg} / 12 \mathrm{~h}$ and i.v. vancomycin 1 $\mathrm{g} / 12 \mathrm{~h}$. Cultured samples of blood, urine and the cath- 

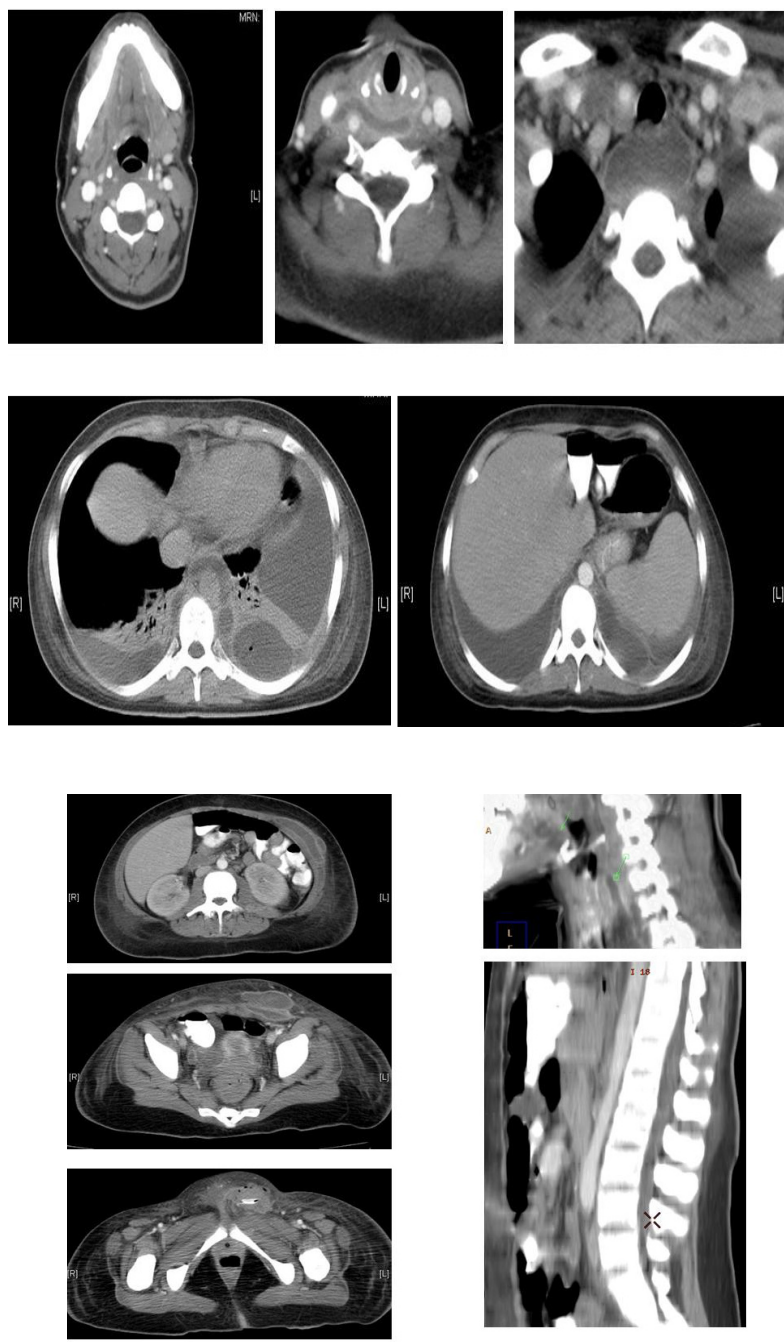

Fig. 2 and 3. Sequence illustrating the cervical, thoracic and abdomino-perineal extension.

eter tip were all negative. There was no trismus or signs of cervical infection.

After 6 days in the ICU the patient was haemodynamically stable with apparent improvement, and was transferred back to the gynaecology ward, where she remained a further 2 days, with fever reaching $38.8^{\circ}$ C. The amikacin was stopped and she was given meropenen $2 \mathrm{~g} / 12 \mathrm{~h}$; she also underwent a second debridement and drainage of the vulvar abscess. Given the absence of improvement, further abdomino-pelvic ultrasound and abdominal contrast-enhanced CT studies were done, which led to completion of the study with chest and cervical CT studies. A report was made of a bilateral pleural collection suggestive of empyema, predominantly on the left, together with condensation of the underlying lung, associated with a collection in the prevertebral space extending from the submandibular / retropharyngeal region to the mediastinum, displacing the oesophagus and airway and which connected caudally as a periaortic tongue with multiple fluid collections following muscular planes from the left hypochondrium to the vulva, and laterally from the right psoas muscle to the infrarenal areas (Figs. 2-3). With these results, which clarified the tortuosity and origin of the symptoms, urgent surgical debridement was performed of the collections by bilateral cervicotomy and right posterolateral thoracotomy, leaving drainage tubes in place. Accordingly, she returned to the ICU for 3 days, where antibiotic treatment was maintained (i.v. meropenem $1 \mathrm{~g} / 8 \mathrm{~h}+$ vancomycin $1 \mathrm{~g} / 12 \mathrm{~h}$ ) together with local dressings and imaging controls that showed clear improvement of the collections. With effect from this point, the patient returned to the ward and had a favourable course. She was discharged 11 days after the cervico-thoracic drainages. Revision one month later showed no findings of note.

\section{Discussion}

The surprising evolution of this patient warrants analysis of those aspects that could have influenced the propagation of the OI. In theory, the patient presented no systemic factor related to immunosuppression, as can occur with chronic steroid or immunsuppressive therapy, or in patients with diabetes, cirrhosis, HIV infection, or other debilitating systemic disorders. She appeared to be immunocompetent, though she did receive methylprednisolone ( $80 \mathrm{mg} / 8 \mathrm{~h}$ in a tapering dose) during her first admission.

The virulence of pathogens based on their biochemical and enzymatic properties could favour the spread of the infection. Unfortunately, no sample was taken for culture and an antibiogram of the alveolar pus at the extraction of tooth 47 , which would have enabled suitable antibiotics from the outset and a correlation with the results of the vulvar sample, which were positive for Escherichia coli, Bacterioides and Streptococcus mitis. No surgical drainage was carried out on the first day of admission, nor on the following days given the good course of the patient after the dental extraction; this, too, would have provided a sample for culture and probably led to an early improvement and cure.

The initial $2 \times 1.6 \mathrm{~cm}$ hypointense CT image showing a phlegmonous aspect reduced the importance of the ultrasound report, which described a suspected $3 \times 4 \mathrm{~cm}$ abscess. In addition, the apparent cure during the first admission should have been confirmed with a control cervical CT prior to discharge.

During the second admission, the patient presented no signs of cervical infection or trismus, with the gynaecological symptoms predominating, which also delayed the diagnosis. The lack of adequate improvement after vulvar drainage indicated the need for abdominal ultrasound and contrast-enhanced CT, tests that showed the abdominal collections and their connection with tho- 
racic and cervical areas by means of the corresponding CT studies.

We cannot rule out the possibility that the bacteraemia was also influential in the implantation and future abscessification of isolated septic foci, although their connection with a descending nature and the history of an odontogenic abscess support the theory of a false initial cure of the cervical abscess and its subsequent spread to lower planes, rather than the bacteraemia causing abscesses above the original focus.

Other authors have already described the passage of pus to the abdomen by descending continuity $(4,8)$, which argues in favour of this rare but possible, and in this case exceptional, event.

Thus, treatment of severe abscessed OI should be based on CT-controlled diagnosis and follow-up (4), and should combine intravenous broad-spectrum antibiotics together with surgical drainage of the purulent collections $(4,9)$. Samples of the drainage material should be taken for culture and an antibiogram, in order to give specific antibiotics if the initial empirical regimen shows resistance. Surgical debridement alone may not be curative if the causative pathogens present resistance to the prescribed antibiotics (10).

\section{References}

References with links to Crossref - DOI

1. Jiménez Y, Bagán JV, Murillo J, Poveda R. Odontogenic infections. Complications. Systemic manifestations. Med Oral Patol Oral Cir Bucal. 2004;9 Suppl:143-7;139-43.

2. Dinatale Papa E. Diseminación de la infección odontogénica. Revisión de la literatura. Acta odontol Venez. 2000; 38:37-43.

3. Colmenero Ruiz C, Labajo AD, Yañez Vilas I, Paniagua J. Thoracic complications of deeply situated serous neck infections. J Craniomaxillofac Surg. 1993;21:76-81.

4. Freeman RK, Vallières E, Verrier ED, Karmy-Jones R, Wood DE. Descending necrotizing mediastinitis: An analysis of the effects of serial surgical debridement on patient mortality. J Thorac Cardiovasc Surg. 2000;119:260-7.

5. Lei WY, Chang WH, Shih SC, Liu CJ, Shih CH. Pyogenic liver abscess with Prevotella species and Fusobacterium necrophorum as causative pathogens in an immunocompetent patient. J Formos Med Assoc. 2009;108:253-7.

6. Chung HY, Sung H, Lee MY, Yoon NS, Lee SG, Suh DJ, et al. A case of bacteremia by Atopobium rimae in a patient with liver cirrhosis. Korean J Lab Med. 2007;27:351-4.

7. Pelizzo G, Missana G, Bisi G. Retroperitoneal abscess from odontogenic infection. Clinical case. Minerva Chir. 1997;52:129-30.

8. Villanueva JC, Bravo A, Segura L, Villalobos E, Almazán JJ. Mediastinitis Necrotizante Descendente Secundaria a Infección Periodontal con Extensión A Pleura y Peritoneo. Reporte de un Caso y Revisión. Medicrit. 2009;6:38-45.

9. Kinzer S, Pfeiffer J, Becker S, Ridder GJ. Severe deep neck space infections and mediastinitis of odontogenic origin: clinical relevance and implications for diagnosis and treatment. Acta Otolaryngol. 2009; 129:62-70.

10. Bux P, Mosca A, Del Prete R, Miragliotta G, Chiaravalle N. The importance of microbiological study in the therapy of odontogenic abscesses. A report of a clinical case. Minerva Stomatol. 1996;45:227-30. 